\title{
Application of the Scattering Bond Graph Methodology for Composite Right/Left Handed Transmission Lines
}

\author{
Islem Salem ${ }^{1}$, Hichem Taghouti ${ }^{2}$, Ahmed Rahmani $^{3}$, Abdelkader Mami $^{4}$ \\ UR-LAPER, University of Tunis El Manar, Tunis, Tunisia, ${ }^{1,2,4}$ \\ CRIStAL, Centrale Lille, Lille, France ${ }^{3}$
}

\begin{abstract}
The approach of the metamaterials based on the theory of transmission lines has an influence on the development of the radiofrequency domain, so the types and techniques of elaboration of these artificial lines are quite diversified. This paper will present two models of Artificial Transmission Line CRLH (Composite Right Left Handed) composed of a combination of resonators OSRR (Open Split Ring Resonators) and OSCRR (Complementary Open Split Ring Resonators). This paper will also show how the Scattering Bond Graph (SBG) study methodology allows to provide electromagnetic simulation results (Scattering parameters, phase response) more conducive to Bond Graph (BG) modeling, and will prove the possibility of having the most perfect line (solved the problem of impedance adaptation) and better understanding of the behavior of radiofrequency systems.
\end{abstract}

Keywords-Scattering Bond Graph (SBG); metamaterials; wave matrix [W]; modelization; transmission line; scattering matrix [S]; CRLH; OSCRR; OSRR

\section{INTRODUCTION}

The development of the communication system imposes the evolution of the modeling and study techniques of hyperfrequency systems. As well as, the exploitation of metamaterials technology [1] (periodic structure characterize by wave propagation with opposite phase and group speed) to play an important role in reducing the size of electronic circuits. Among the applications used by this technology are the waveguide structures (filter) and the radiant devices (antenna) which seems to have more advantage compared to the old structures.

Despite the many advantages of the metamatrials techniques [2], the analysis of microwave circuits remains difficult and has problems especially adaptation and modeling that requires manufacturers to redo the complete study to change some parameters which plays a role negative role on the effectiveness of the results obtained.

In [3] and [4], the CRLH (Composite Right Left Handed) structures were introduced by the classical technique which possesses indeterminate equations with several unknowns, thus the resolution of non-homogeneous differential equations.

Since the appearance of the bond graph theory [5] research in the field of dynamic physical systems has been developed, but this theory has remained limited only for low frequency systems. Until 2009 [6], where researchers have developed an analytical method that makes it possible to classify two-port network (at only one lumped element) in microwave, we apply the conjunction between the scattering formalism and the bond graph approach.

In this study, two types of CRLH transmission line structures inspired by the combination of OCSRR (Complementary Open Split Ring Resonators) and OSRR (Open Split Ring Resonators), will be presented and studied and analyzed with the Scattering Bond Graph methodology.

The first part is dedicated to the modulation and deduction of the parameters of the different system circuits by the scattering bond graph method.

The second part is reserved for the development of two new CRLH transmission line structures, and to study the physical characteristics of each structure from the [W] wave matrix. Where this matrix describes the relations between the incident waves and reflects whatever the complexity of the system.

Finally, the reliability of the methodology is determined from the level of adaptation of the transmission lines and the total transfer of the incident waves

\section{SCATTERING BOND MODELING}

The bond graph tool appeared for the first time by Pynter [5], which is a unified graphical language for all domains. This approach is based on the phenomenon of energy exchange (flow and effort) in the system that allows to model and simulate systems that can be multidisciplinary.

The concept of the scattering matrix (generally noted [S]) which was invented for the first time by Wheeler [7], is the study of the n-pole hyper frequency linear system through the parameters of this matrix $S_{i j}$

The conjunction between the two approaches gives birth to a new methodology called Scattering Bond Graph [8], this methodology proved its efficiency of modelling of the microwave system [9]. This method goes through three phases, first is to model the system by the bond graph approach and apply the causality (notion allows to highlight the cause-effect relationship (flow and effort) of the system and to release an associated mathematical model). Secondly 
find the parameters of the wave matrix (denoted [W]) (each subsystem has a wave matrix). Third, find the scattering matrix [S] by applying (1) [7].

$[S]=\left[\begin{array}{cc}\frac{W_{11}}{W_{22}} & \frac{1}{W_{22}}\left(W_{22} W_{11}-W_{21} W_{12}\right) \\ \frac{1}{W_{22}} & \frac{-W_{21}}{W_{22}}\end{array}\right]$

The SBG technique is based on the translation of the electrical circuit based in lumped elements into a graphic diagram [10]. But in radio frequency each element admits its own electrical and magnetic characteristics (electric and magnetic fields). In the case of study with the BG approach we can consider that the whole system is a resonant element, which allows us to have more precision.

As shown in Fig. 1, the circuit in " $\pi$ " is composed by the combination of a shunt admittance " $Y$ " in parallel with a series impedance " $Z$ " in parallel with another shunt admittance "Y".

Using the bond-graph approach definitions, the " $\pi$ " causal circuit model (present of a causal link between a potential input and output of the model) can be represented by Fig. 2 (0-1-0 junction).

From the causal model bond graph 0-1-0 junction we can directly extract the wave matrix $[\mathrm{W}]_{010}$

$[W]_{010}=$

$\frac{1}{2}\left[\begin{array}{cc}z y^{2}+2 y z+2 y+z+2 & z y^{2}-z+2 y \\ -z y^{2}+z-2 y & -z y^{2}+2 y z-2 y-z+2\end{array}\right]$

As shown in Fig. 3 the circuit in "T" is composed by the combination of a series impedance " $Z$ " in parallel with a shunt admittance " $Y$ " in parallel with another series impedance " $Z$ " [9] [11].

Using the bond-graph approach definitions, the " $\mathrm{T}$ " causal circuit model can be represented by Fig. 4 (1-0-1 junction).

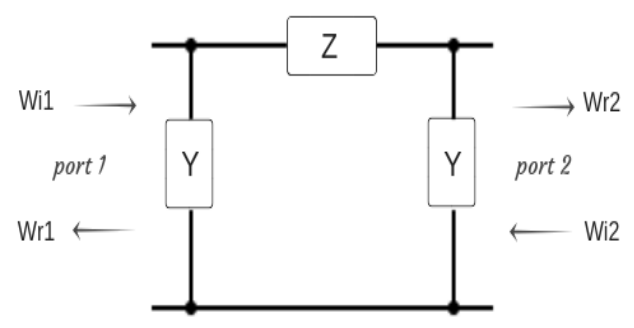

Fig. 1. Model of a Circuit in " $\pi$ ".

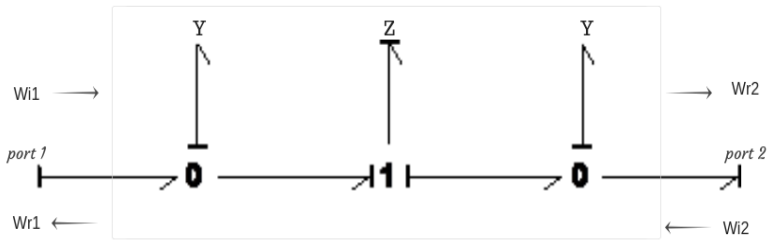

Fig. 2. Causal Bond Graph Model of Circuit in " $\pi$ ".

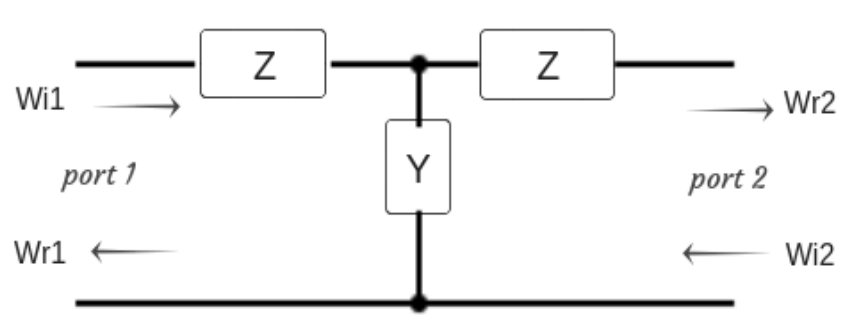

Fig. 3. Model of a Circuit in "T".

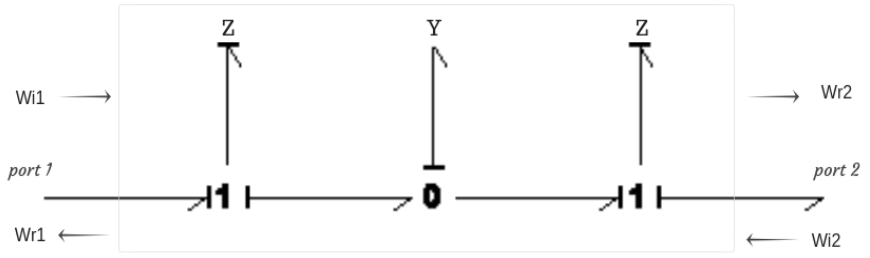

Fig. 4. Causal Bond Graph Model of Circuit in "T".

From the causal model bond graph 0-1-0 junction can directly extract the wave matrix $[\mathrm{W}]_{101}$

$[W]_{101}=$

$\frac{1}{2}\left[\begin{array}{cc}y z^{2}+2 y z+2 z+y+2 & y z^{2}+y-2 z \\ y z^{2}-y+2 z & -y z^{2}+2 y z-2 z-y+2\end{array}\right]$

\section{ANALYSIS COMPOSITE RIGHT/LEFT HANDED (CRLH) TRANSMISSION LINE BY SCATTERING BOND GRAPH METHODOLOGY}

The CRLH line introduced for the first time by the American Cloz in 2003 [12], and as its name indicates, the CRLH is transmission line has a right handed wave propagation (admits a positive permittivity and permeability $\left(\varepsilon_{\mathrm{r}}>0\right.$ and $\left.\mu_{\mathrm{r}}>0\right)$ ), but also a wave propagation left handed (also called artificial doubly negative materials $\left(\varepsilon_{\mathrm{r}}<0\right.$ and $\mu_{\mathrm{r}}<$ $0)$ ). Several researches have shown that the exploitation of CRLH has the advantage of being able to reduce the size and the number of the electrical components for the systems of wireless communication.

In the rest of this study, will be interested in the development of the CRLH transmission line modeling technique based on the combination of the OSRR resonator introduced by J. Martel and Al in 2004 [13] and OCSRR resonator, produced by A.Velz and $\mathrm{Al}$ in 2009 [14]. This contribution will be made by the proposed methodology Scattering Bond Graph.

The CRLH line is composed of three sub-systems in cascade, from which the choice of the SBG approach wasn't arbitrary since among the advantages of this methodology it solved the problem of adaptation between subsystems, in other words will haven't to redo the calculations or add parameters to reach the adaptation between the different subsystems (OSRR and OCSRR resonators) before having the adaptation to the complete system (CRLH line). 
The solution is to apply the product of the wave matrices [W] extract directly from the equivalent diagram Bond Graph before finding the scattering parameter (through the relation (1))

The first sub-system is the OSRR resonator. Fig. 5 represents its equivalent Bond Graph model (circuit T) where it is composed by a capacitance element $\mathrm{C}$ (parasitic capacitance will be taken into account in the design) in serie (1-junction) with $\mathrm{C}_{\text {serie }}$ and $\mathrm{L}_{\text {serie }}$, then in parallel with a second capacitance element $\mathrm{C}$.

By applying the laws of the BG approach and causality obtain the wave matrix $[\mathrm{W}]$ OSRR

$[\mathrm{W}]_{\text {OSRR }}=$

$\frac{1}{2}\left[\begin{array}{cc}z_{s} y^{2}+2 y z_{s}+2 y+z_{s}+2 & z_{s} y^{2}-z_{s}+2 y \\ -z_{s} y^{2}+z_{s}-2 y & -z_{s} y^{2}+2 y z_{s}-2 y-z_{s}+2\end{array}\right]$

Where, $z_{s}$ and $y$ represent respectively the normalized series impedance and the normalized admittance (s: represents the Laplace operator).

$z_{s}=\left(\frac{1}{\tau_{c_{\text {serie }}}}+\tau_{L_{\text {serie }}}\right) s$

$y=\tau_{c} s$

The second sub-system is the OCSRR resonator. Fig. 6 represents its equivalent Bond Graph model (circuit $\pi$ ) where it is composed by an inductance element $\mathrm{L}$ (parasitic inductance will be taken into account in the design) in parallel (0-junction) with $\mathrm{C}_{\text {parallel }}$ and $\mathrm{L}_{\text {parallel}}$, in series with a second inductance element $\mathrm{L}$.

By applying the laws of the BG approach and causality obtain the wave matrix $[\mathrm{W}]$ OCSRR

$[W]_{\text {OCSRR }}=$

$\frac{1}{2}\left[\begin{array}{cc}y_{p} z^{2}+2 y_{p} z+2 z+y_{p}+2 & y_{p} z^{2}+y_{p}-2 z \\ y_{p} z^{2}-y_{p}+2 z & -y_{p} z^{2}+2 y_{p} z-2 z-y_{p}+2\end{array}\right]$
Where, $\mathrm{y}_{\mathrm{p}}$ and $\mathrm{z}$ represent respectively the normalized parallel admittance and the normalized impedance.

$y_{p}=\left(\frac{1}{\tau_{\text {Larallel }}}+\tau_{C_{\text {parallel }}}\right) s$

$Z=\tau_{L} S$

\section{A. OCSRR-OSRR-OCSRR Line Model}

The first proposal for a CRLH transmission line is represented by Fig. 7 where it consists of two OCSRR resonators and an OSRR resonator that are cascading.

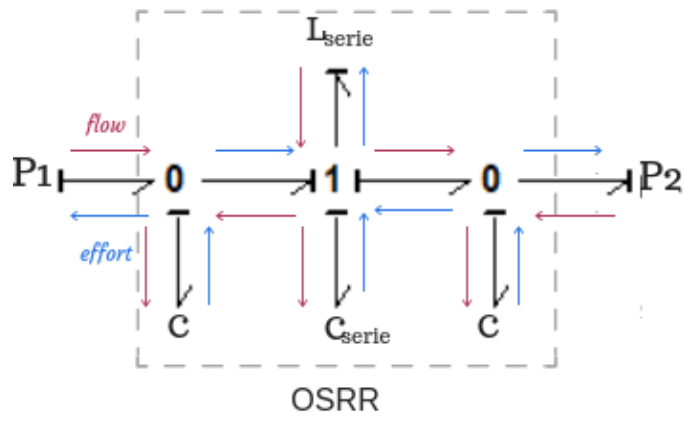

Fig. 5. Representation of the Model by Bond Graph of the Resonator OSRR.

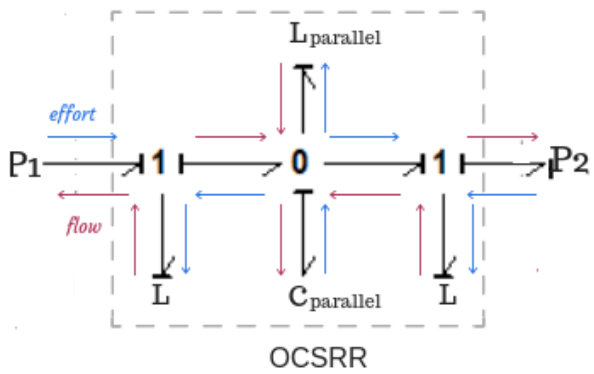

Fig. 6. Representation of the Model by Bond Graph of the Resonator OCSRR.

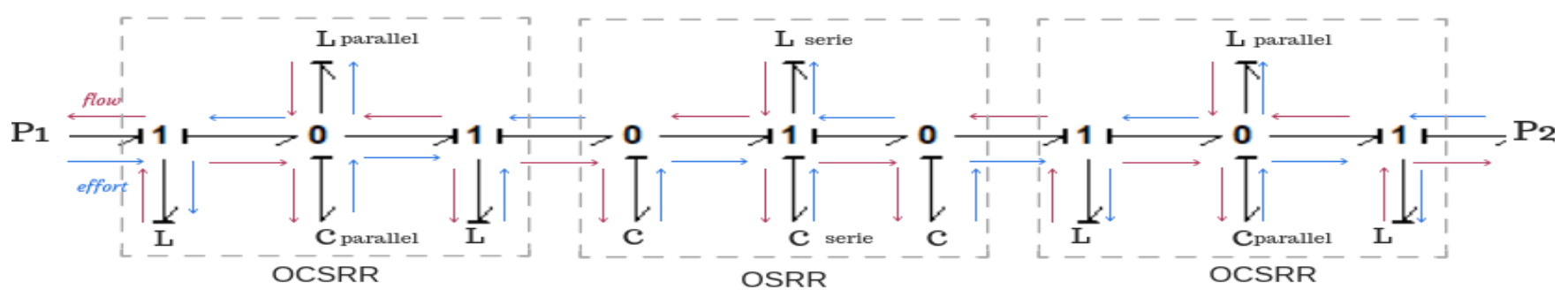

Fig. 7. Representation of the Model by Bond Graph of the CRLH Line.

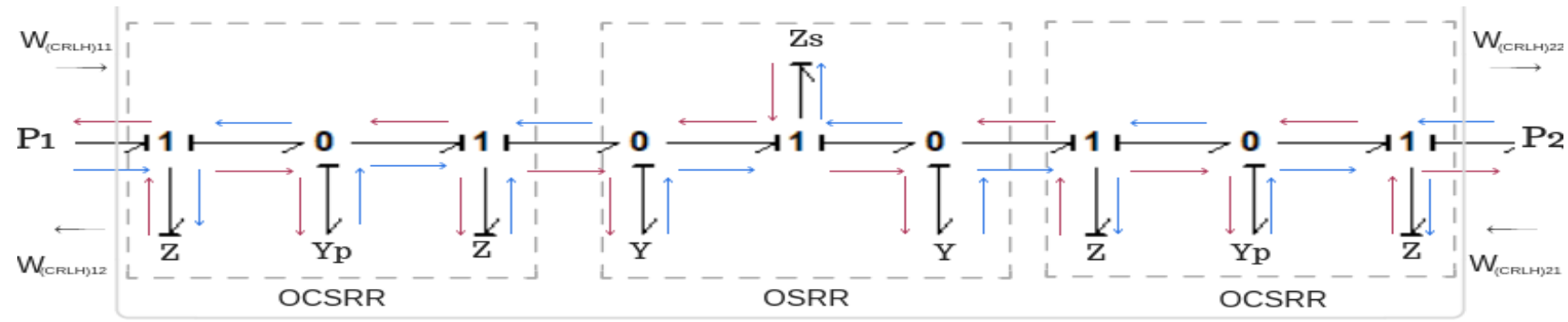

Fig. 8. Representation of the Reduced Model by Bond Graph of the CRLH Line. 
By applying the properties of the bond graph approach to the representation of CRLH, the reduced model is presented by Fig. 8 .

The wave matrix $\left[\mathrm{W}_{\mathrm{CRLH}}\right]_{\mathrm{A}}$ of the CRLH line can be written as follows (by (4) and (6)).

$\left[\mathrm{W}_{\mathrm{CRLH}}\right]_{\mathrm{A}}=[\mathrm{W}]_{\mathrm{OCSRR}} *[\mathrm{~W}]$ OSRR $*[\mathrm{~W}]$ OCSRR

$[\mathrm{WCRLH}]_{A}=\left(\begin{array}{ll}\mathrm{WCRLH}_{11 A} & \mathrm{WCRLH}_{12 A} \\ \mathrm{WCRLH}_{21 A} & \mathrm{WCRLH}_{22 A}\end{array}\right)$

WCRLH11A $=\frac{1}{8}(-(y 2 z s+2 y-z s)(y p z 2+y p-2 z)+$

$(y 2 z s+2 y z s+2 y+z s+2)(y p z 2+2 y p z+y p+2 z+2))$

$(y p z 2+2 y p z+y p+2 z+2)-(-(y 2 z s+2 y-z s)(y p z 2+2 y p z+y p+2 z+2)$

$+(y p z 2+y p-2 z)(y 2 z s-2 y z s+2 y+z s-2))(y p z 2-y p+2 z)$

$\mathrm{W}_{\mathrm{CRLH} 12 \mathrm{~A}}=-\frac{1}{8}\left(\left(y^{2} z_{s}+2 y-z_{s}\right)\left(y_{p} z^{2}+y_{p}-2 z\right)-\left(y^{2} z_{s}+2 y z_{s}+2 y+z_{s}+2\right)\right.$ $\left.\left(y_{p} z^{2}+2 y_{p} z+y_{p}+2 z+2\right)\right)\left(y_{p} z^{2}+y_{p}-2 z\right)+\left(-\left(y^{2} z_{s}+2 y-z_{s}\right)\left(y_{p} z^{2}+2 y_{p} z+y_{p}+2 z+2\right)\right.$ $\left.+\left(y_{p} z^{2}+y_{p}-2 z\right)\left(y^{2} z_{s}-2 y z_{s}+2 y+z_{s}-2\right)\right)\left(y_{p} z^{2}-2 y_{p} z+y_{p}+2 z-2\right)$

$\mathrm{W}_{\mathrm{CRLH} 21 \mathrm{~A}}=\frac{1}{8}\left(\left(y^{2} z_{s}+2 y-z_{s}\right)\left(y_{p} z^{2}-y_{p}+2 z\right)+\left(y^{2} z_{s}-2 y z_{s}+2 y+z_{s}-2\right)\right.$ $\left.\left.\left(y_{p} z^{2}-2 y_{p} z+y_{p}+2 z-2\right)\right) y_{p} z^{2}-y_{p}+2 z\right)+\left(\left(y^{2} z_{s}+2 y-z_{s}\right)\right.$

$\left.\left(y_{p} z^{2}-2 y_{p} z+y_{p}+2 z-2\right)+\left(y_{p} z^{2}-y_{p}+2 z\right)\left(y^{2} z_{s}+2 y z_{s}+2 y+z_{s}+2\right)\right)\left(y_{p} z^{2}+2 y_{p} z+y_{p}+2 z\right.$ $+2)$

$\mathrm{W}_{\mathrm{CRLH} 22 \mathrm{~A}}=-\frac{1}{8}\left(\left(y^{2} z_{s}+2 y-z_{s}\right)\left(y_{p} z^{2}-y_{p}+2 z\right)+\left(y^{2} z_{s}-2 y z_{s}+2 y+z_{s}-2\right)\right.$ $\left.\left(y_{p} z^{2}-2 y_{p} z+y_{p}+2 z-2\right)\right)\left(y_{p} z^{2}-2 y_{p} z+y_{p}+2 z-2\right)+\left(\left(y^{2} z_{s}+2 y-z_{s}\right)\right.$ $\left.\left(y_{p} z^{2}-2 y_{p} z+y_{p}+2 z-2\right)+\left(y_{p} z^{2}-y_{p}+2 z\right)\left(y^{2} z_{s}+2 y z_{s}+2 y+z_{s}+2\right)\right)\left(y_{p} z^{2}+y_{p}-2 z\right)$

According to the equivalent diagram of the assembly of the three proposed resonators, have obtained the characteristic wave matrix $\left[\mathrm{W}_{\mathrm{CRLH}}\right]_{\mathrm{A}}$. To obtain the electromagnetic responses of this system, it is necessary to directly deduce the scattering matrix $\left[\mathrm{S}_{\mathrm{CRLH}}\right]_{\mathrm{A}}$ from the whole system.

$\left[S_{C R L H}\right]_{A}=\left[\begin{array}{lc}\frac{W_{\mathrm{CRLH} 11 A}}{W_{\mathrm{CRLH} 2 A}} & \frac{\left(W_{\mathrm{CRLH} 22 A} W_{\mathrm{CRLH} 11 A}-W_{\mathrm{CRLH} 21 A} W_{\mathrm{CRLH} 12 A}\right)}{W_{\mathrm{CRLH} 22}} \\ \frac{1}{W_{\mathrm{CRLH} 22 A}} & \frac{-W_{\mathrm{CRLH} 21 A}}{W_{\mathrm{CRLH} 22 A}}\end{array}\right]$

Fig. 9 represents the frequency response of the proposed $\mathrm{CRLH}_{\mathrm{A}}$ line (transmission and reflection coefficient) and Fig. 10 represents the phase response.

According to the result of the simulation obtained, the $\mathrm{CRLH}_{\mathrm{A}}$ admits two spades of frequencies at resonance frequencies $2.2 \mathrm{GHz}$ and $3.8 \mathrm{GHz}$ and at most $-23 \mathrm{~dB}$, which proves that the system is adapted to a bandwidth of $3.4 \mathrm{GHz}$. But the incident wave isn't totally transmitted.

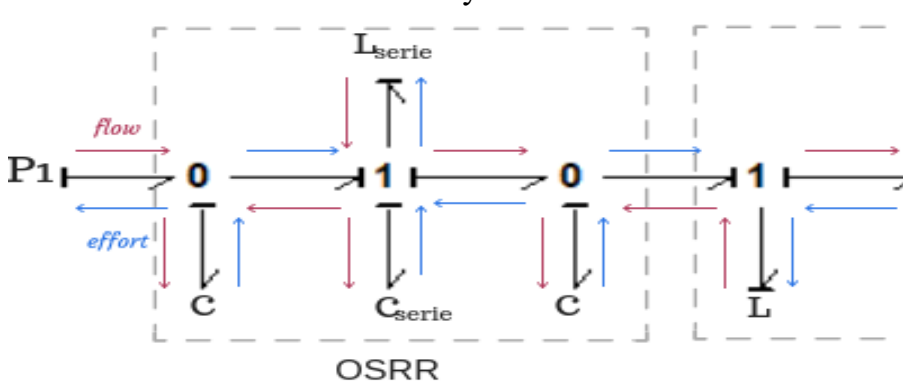

\section{B. OSRR-OCSRR-OSRR Line Model}

The second proposal for a CRLH transmission line is represented by Fig. 11 where it consists of two OSRR resonators and an OCSRR resonator that are cascading.

By applying the properties of the bond graph approach to the representation of CRLH, the reduced model is presented by Fig. 12 .

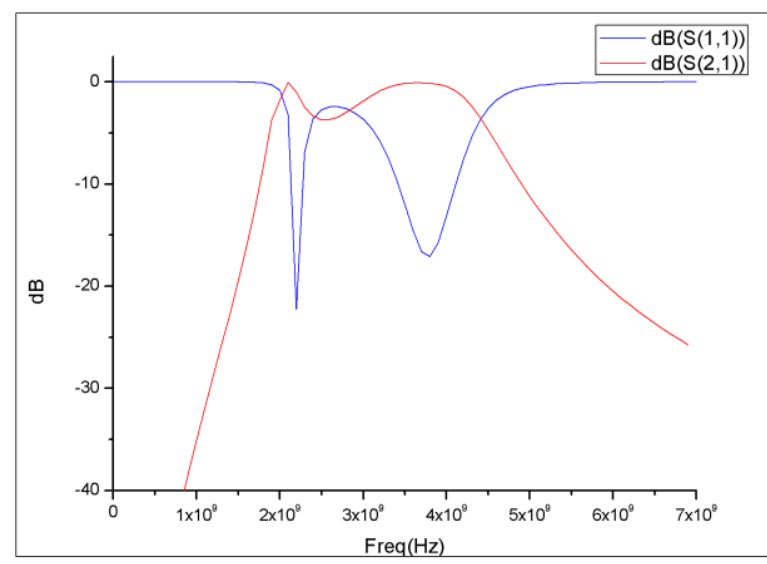

Fig. 9. The Frequency Response of the Proposed CRLH $\mathrm{CL}_{\mathrm{A}}$.

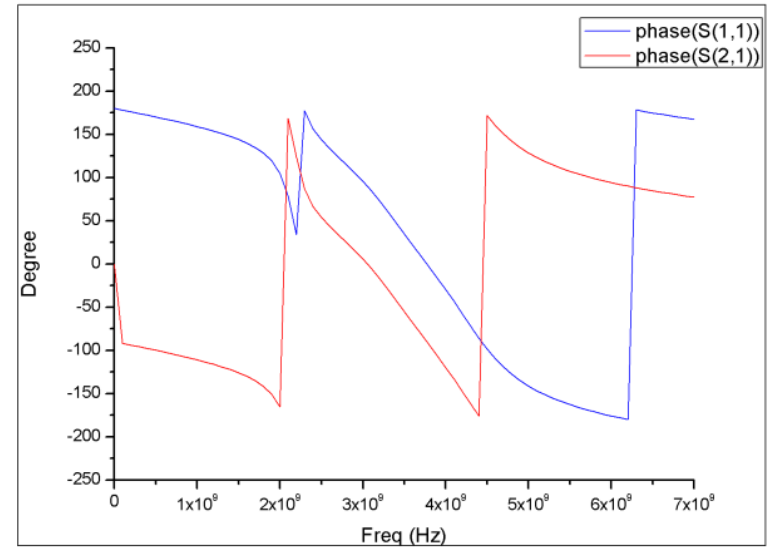

Fig. 10. The Phase Response.

Fig. 11. Representation of the $\mathrm{CRLH}_{\mathrm{B}}$ Model by Bond Graph. 


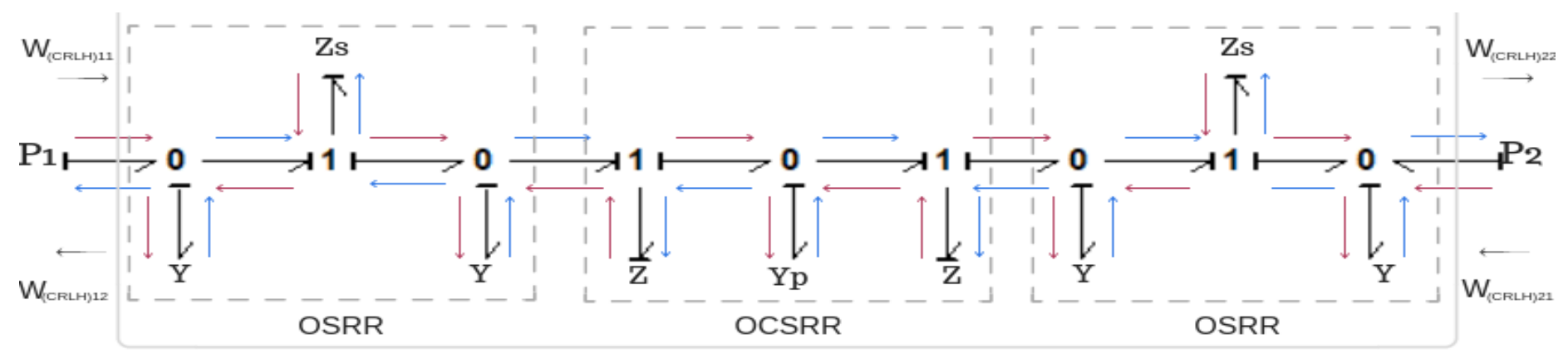

Fig. 12. Representation of the $\mathrm{CRLH}_{\mathrm{B}}$ Reduced Model by Bond Graph.

The wave matrix $\left[\mathrm{W}_{\mathrm{CRLH}}\right]_{\mathrm{A}}$ of the CRLH line can be written as follows (by (4) and (6)):

$\left[\mathrm{W}_{\mathrm{CRLH}}\right]_{\mathrm{B}}=[\mathrm{W}]_{\mathrm{OCSRR}} *[\mathrm{~W}]_{\mathrm{OSRR}} *[\mathrm{~W}]_{\mathrm{OCSRR}}$

$[\mathrm{WCRLH}]_{B}=\left(\begin{array}{ll}\mathrm{WCRLH}_{11 B} & \mathrm{WCRLH}_{12 B} \\ \mathrm{WCRLH}_{21 B} & \mathrm{WCRLH}_{22 B}\end{array}\right)$

$\left[\mathrm{W}_{\mathrm{CRLH}}\right]_{11 \mathrm{~B}}=\frac{1}{8}\left(\left(y^{2} z_{s}+2 y-z_{s}\right)\left(y_{p} z^{2}-y_{p}+2 z\right)+\left(y^{2} z_{s}+2 y z_{s}+2 y+z_{s}+2\right)\right.$ $\left.\left(y_{p} z^{2}+2 y_{p} z+y_{p}+2 z+2\right)\right)\left(y^{2} z_{s}+2 y z_{s}+2 y+z_{s}+2\right)-\left(-\left(y^{2} z_{s}+2 y-z_{s}\right)\left(y_{p} z^{2}-2 y_{p} z+y_{p}+\right.\right.$ $\left.2 z-2)+\left(y_{p} z^{2}+y_{p}-2 z\right)\left(y^{2} z_{s}+2 y z_{s}+2 y+z s+2\right)\right)\left(y^{2} z_{s}+2 y-z_{s}\right)$

$\left[\mathrm{W}_{\mathrm{CRLH}}\right]_{12 \mathrm{~B}} \frac{1}{8}\left(\left(y^{2} z_{s}+2 y-z s\right)\left(y_{p} z^{2}-y_{p}+2 z\right)+\left(y^{2} z_{s}+2 y z_{s}+2 y+z s+2\right)\right.$ $\left.\left(y_{p} z 2+2 y_{p} z+y_{p}+2 z+2\right)\right)\left(y^{2} z_{s}+2 y-z_{s}\right)-\left(-\left(y^{2} z_{s}+2 y-z_{s}\right)\left(y_{p} z^{2}-2 y_{p} z+y_{p}+2 z-2\right)+(\right.$ $\left.\left.y_{p} z^{2}+y_{p}-2 z\right)\left(y^{2} z_{s}+2 y z_{s}+2 y+z_{s}+2\right)\right)\left(y^{2} z_{s}-2 y z_{s}+2 y+z_{s}-2\right)$

$\left[\mathrm{W}_{\mathrm{CRLH}}\right]_{21 \mathrm{~B}}=\frac{1}{8}\left(\left(y^{2} z_{s}+2 y-z_{s}\right)\left(y_{p} z^{2}+y_{p}-2 z\right)-\left(y^{2} z_{s}-2 y z_{s}+2 y+z_{s}-2\right)\right.$ $\left.\left(y_{p} z^{2}-2 y_{p} z+y_{p}+2 z-2\right)\right)\left(y^{2} z_{s}+2 y-z_{s}\right)-\left(\left(y^{2} z_{s}+2 y-z_{s}\right)\left(y_{p} z^{2}+2 y_{p} z+y_{p}+2 z+2\right)\right.$ $\left.+\left(y_{p} z^{2}-y_{p}+2 z\right)\left(y^{2} z_{s}-2 y z_{s}+2 y+z_{s}-2\right)\right)\left(y^{2} z_{s}+2 y z_{s}+2 y+z_{s}+2\right)$

$\left[\mathrm{W}_{\mathrm{CRLH}}\right]_{22 \mathrm{~B}}=\frac{1}{8}\left(\left(y^{2} z_{s}+2 y-z_{s}\right)\left(y_{p} z^{2}+y_{p}-2 z\right)-\left(y^{2} z_{s}-2 y z_{s}+2 y+z_{s}-2\right)\right.$ $\left.\left(y_{p} z^{2}-2 y_{p} z+y_{p}+2 z-2\right)\right)\left(y^{2} z_{s}-2 y z_{s}+2 y+z_{s}-2\right)-\left(\left(y^{2} z_{s}+2 y-z_{s}\right)\left(y_{p} z^{2}+2 y_{p} z+y_{p}+2\right.\right.$ $\left.z+2)+\left(y_{p} z^{2}-y_{p}+2 z\right)\left(y^{2} z_{s}-2 y z_{s}+2 y+z_{s}-2\right)\right)\left(y^{2} z_{s}+2 y-z_{s}\right)$

According to the equivalent diagram of the assembly of the three proposed resonators, have obtained the characteristic wave matrix $\left[\mathrm{W}_{\mathrm{CRLH}}\right]_{\mathrm{B}}$. To obtain the electromagnetic responses of this system, it is necessary to directly deduce the scattering matrix $\left[\mathrm{S}_{\mathrm{CRLH}}\right]_{\mathrm{B}}$ from the whole system.

$\left[\mathrm{S}_{\mathrm{CRLH}}\right]_{\mathrm{B}}=$

$\left[\begin{array}{lc}\frac{W_{\mathrm{CRLH} 11 B}}{W_{\mathrm{CRLH} 22 B}} & \left(W_{\mathrm{CRLH} 22 B} W_{\mathrm{CRLH} 11 B}-W_{\mathrm{CRLH} 21 B} W_{\mathrm{CRLH} 12 B}\right) \\ \frac{1}{W_{\mathrm{CRLH} 22 B}} & \frac{-W_{\mathrm{CRLH} 21 B}}{W_{\mathrm{CRLH} 22 B}}\end{array}\right]$

Fig. 13 represents the frequency response of the proposed $\mathrm{CRLH}_{\mathrm{B}}$ line (transmission and reflection coefficient) and Fig. 14 represents the phase response.

According to the result of the simulation obtained, the $\mathrm{CRLH}_{\mathrm{B}}$ admits two spades of frequencies at resonance frequencies $2.1 \mathrm{GHz}$ and $4.4 \mathrm{GHz}$ and at most $-35 \mathrm{~dB}$, which proves that the system is adapted to a bandwidth of $3.4 \mathrm{Ghz}$. And the incident wave is totally transmitted.

\section{Discussion}

The choice of the most perfect model type is made according to the highest adaptation. This model must also consider the need to have the minimum loss.

It is noted that the line $\mathrm{CRLH}_{\mathrm{B}}$ is more preferment than the line $\mathrm{CRLH}_{\mathrm{A}}$. Because according to the results of simulation the line $\mathrm{CRLH}_{\mathrm{B}}$ is more adaptable is possesses a wider bandwidth and does not admit of loss. So for the realization of line CRLH one proposes the second solution.

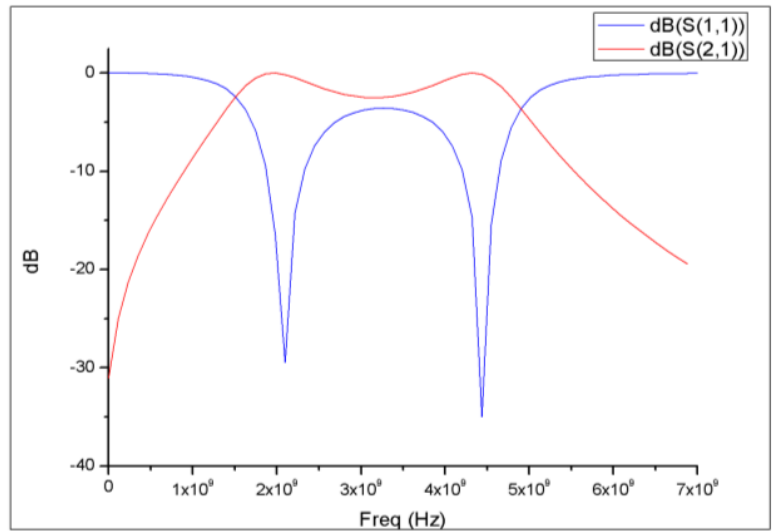

Fig. 13. The Frequency Response of the Proposed CRLH $\mathrm{H}_{\mathrm{B}}$

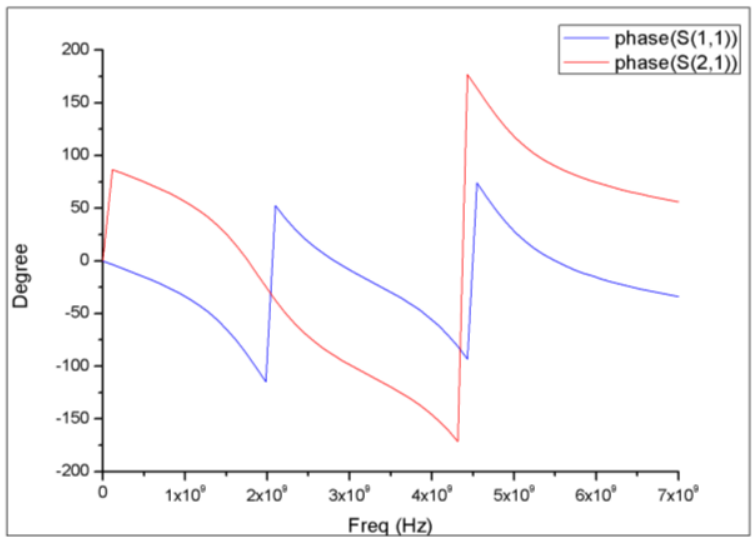

Fig. 14. The Phase Response. 


\section{CONCLUSION}

In the first part, we modeled the equivalent circuits of the resonators OCSRR and OSRR, and then we developed the wave matrix [W] by applying the properties of the bond graph.

In the second part, we proposed two new CRLH transmission line structures based on the combination of OCSRR and OSRR.

In the third part, we analyzed the characteristics of each transmission line from its scattering parameters deduced from the diagram bong graph.

Finally, we proved by simulation that the scattering bond graph methodology is a useful tool to check the performance of radiofrequency models before the implementation phase. We also validated that SBG is a simpler and more reliable technique for complex communication systems.

In the study of the future, we use the efficiency of the scattering bond graph methodology in the study of the more complex system that is the Dual Composite Right/Left Handed Transmission.

\section{REFERENCES}

[1] Grbic, G. V Eleftheriades, A. Grbic, and G. V Eleftheriades, "Experimental verification of backward-wave radiation from a negative refractive index metamaterial Experimental verification of backwardwave radiation from a negative refractive index metamaterial," vol. 5930, 2002.

[2] W. Hong, B. Liu, Y. Wang, Q. Lai, and H. Tang, "Half Mode Substrate Integrated Waveguide: A New Guided Wave Structure for Microwave and Millimeter Wave Application," vol. 152, p. 4244, 2006.

[3] A. A. Chaudhry, J. K. Arif, Z. Ahmed, M. A. Chaudhary, and M. Bin Ihsan, "Parameter Extraction of Composite Right / Left Handed ( CRLH
) Transmission Line Unit Cell Using Off Resonance Method," pp. 779781, 2017.

[4] P. Fathi, Z. Atlasbaf, and K. Forooraghi, "Compact Dual-Wideband Bandpass Filter Using CSRR Based Extended Right / Left-Handed Transmission Line," vol. 81, no. January, pp. 21-30, 2018.

[5] H. Paynter and M. M. I. T. Press, "Analysis and design of engineering systems: class notes for M. I . T . course 2.751/by Henry M . Paynter; with the assistance of Peter Briggs . not subject to copyright . Users are free to copy, use," 1961.

[6] H. Taghouti, A. Mami, and B. Graph, "Extraction , Modelling and Simulation of the Scattering Matrix of a Chebychev Low-Pass Filter with cut-off frequency $100 \mathrm{MHz}$ from its Causal and Decomposed Bond Graph Model," vol. 10, no. 1, pp. 29-37, 2010.

[7] "Constructing the scattering matrix for optical microcavities as a nonlocal boundary value problem," vol. 5, no. 6, pp. 20-28, 2017.

[8] S. Dridi, I. Ben Salem, and L. El Amraoui, "A Multi-Energetic Modeling Approach based on Bond Graph Applied to In-Wheel-Motor Drive System," vol. 9, no. 10, pp. 422-429, 2018.

[9] I. Salem, H. Taghouti, and A. Mami, "Modeling CSRR and OCSRR loaded transmission line by bond graph approach methodology," 2018 9th Int. Renew. Energy Congr. IREC 2018, no. Irec, pp. 1-6, 2018.

[10] J. Sabri, N. Omrane, T. Hichem, M. Abdelkader, and G. Ali, "Miniaturized Meander Slot Antenna Tor RFID TAG with Dielectric Resonator at $60 \mathrm{Ghz}$," Int. J. Adv. Comput. Sci. Appl., vol. 7, no. 4, pp. 373-380, 2016.

[11] S. Khmailia, "Impedance Matching of a Microstrip Antenna," vol. 8, no. 7, pp. 19-23, 2017.

[12] F. S. Paper, C. Caloz, T. Itoh, and L. Angeles, “TU2C-3 Invited - Novel Microwave Devices and Structures Based on the Transmission Line Approach of Meta-Materials," pp. 195-198, 2003.

[13] J. Martel et al., "A New LC Series Element for Compact Bandpass Filter Design," vol. 14, no. 5, pp. 210-212, 2004.

[14] A. Vélez et al., "Open Complementary Split Ring Resonators ( OCSRRs ) and Their Application to Wideband CPW Band Pass Filters," vol. 19, no. 4, pp. 197-199, 2009. 